\title{
Effectiveness assessment of vaccination policy against measles epidemic in Japan using an age-time two-dimensional mathematical model
}

\author{
Yusuke Maitani · Hirofumi Ishikawa
}

Received: 14 February 2011/Accepted: 8 April 2011/Published online: 7 May 2011

(C) The Japanese Society for Hygiene 2011

\begin{abstract}
Objectives In 2007, measles prevailed among the youth and young adult population in Japan, creating in a serious social problem. Among the developed countries, Japan has a relatively high incidence of measles. The objective of this study was to assess the effect of improvements in the vaccination policy against measles through simulations.

Methods We developed an age-time two-dimensional model for the transmission of measles to reflect an age structure, enabling contact rate to be selected by age. Introduction of the maternal immunity class into the model allowed natural infection and vaccination to be discriminated along the course of an infant's mother acquiring the immunity, thereby resulting in an improved accuracy of the simulations in infants. Several vaccination scenarios were attempted in order to assess the influence of various vaccination policies on the prevention of a measles epidemic. Results The results of this quantitative study indicated that suppression of a measles outbreak requires the maintenance of high vaccine coverage and that a decline in vaccine coverage may result in a measles epidemic.

Conclusions The present standard immunization program for measles will maintain an acceptable level of immunity and is therefore associated with a low risk of an epidemic after discontinuation of the third and fourth stages as scheduled-as long as at least $90 \%$ vaccine coverage of the first and second is maintained. The simulation results show that discontinuation of the third and fourth stages of vaccination as scheduled should be accompanied by endeavors
\end{abstract}

Y. Maitani · H. Ishikawa $(\bowtie)$

Department of Human Ecology,

Graduate School of Environmental Science,

Okayama University, Okayama 700-8530, Japan

e-mail: ishikawa@ems.okayama-u.ac.jp to maintain appropriate high vaccine coverage of the first and second stages.

Keywords Measles - Vaccination coverage · Routine immunization program · Age-time model . Maternal immunity

\section{Introduction}

In 2007, measles prevailed among the youth and young adult (teenagers and those in their 20s) population of Japan, creating serious social problems, such as closure of college/ university classes. The incidence of measles in Japan has been reported to be 10,000-30,000 per year [1], but the actual incidence is estimated to be about tenfold higher than the number of reported cases [1]. The aim of this study was to analyze the effect of improvements to the standard vaccination program for measles, with a special focus on the coverage of measles vaccination. Vaccination against measles was incorporated into the national immunization program in Japan in 1978, but in 1994 some children had an allergic reaction due to sensitization to the gelatin contained in the measles-mumps-rubella vaccine (MMR). As a consequence, the measles vaccination policy was modified, i.e., from mass immunization in schools to individual immunization, and from obligatory to voluntary immunization. This policy change has led to the ongoing low vaccination coverage against measles that was immediately detectable [1]. Because it is feared that an outbreak of measles will occur under these circumstances, a second stage of measles vaccination was introduced into the routine immunization program in 2006. In 2008, a third and fourth stage of measles vaccination were later added to the routine immunization program for a limited period of 
5 years (2008-2012). The first to fourth stages of vaccination were targeted at infants ( 1 year old), children 1 year prior to starting elementary school (5-6 years old), students in the first grade of junior high school (12-13 years old), and students in the third grade of high school (17-18 years old), respectively [2]. Those autonomous local and regional authorities that achieved high vaccination coverage in the second stage did so by encouraging individual vaccination, establishing a strong partnership with institutions, distributing leaflets, mailing postcards, posting notifications of vaccination on city bulletin boards and posters, and developing a system to target non-vaccinated individuals, among others (Niigata, Odawara, Takamatsu, and Kurashiki cities, and Fukui and Akita prefectures) [3-8]. Those authorities that achieved high vaccination coverage in the third and the fourth stages had a program that involved notification of individuals for vaccination, cooperation with schools, and mass immunization against measles, among others (Johetsu, Tsukuba, Hakodate, and Hamamatsu cities) [9-12].

The World Health Organization (WHO) assesses the numbers of measles patients and deaths attributable to measles to be more than $30,000,000$ and 875,000 each year, respectively [1]. In most developed countries, including the USA and Korea, the law obliges parents to present a certificate of measles vaccination when their child enters elementary school [13], which has resulted in a high vaccination coverage. Consequently, any outbreaks of measles in such countries mainly occur among immigrants from regions where measles is epidemic.

In recent years, most individuals have acquired immunity to measles by vaccination. Because the antibody titer acquired by vaccination is low compared with that by natural infection, the possibility of re-infection with measles emerges in teenagers and in young adults in their 20s [14].

There have been several studies on a mathematical model for the transmission of measles [15-20]. In this study, we developed an age-time two-dimensional model for the transmission of measles to reflect an age structure, thereby enabling the selection of contact rate by age. We analyzed vaccination policy and the influence of vaccination coverage through model simulations. To make the model more precise, we developed the model to be able to distinguish maternal immunity derived from a mother who acquired immunity by natural infection from that derived from a mother who acquired immunity by vaccination because most mothers have acquired the antibody against measles by vaccination in recent years. We also used epidemiological classes comprising individuals with low antibody titer so as to be able to estimate the change in the number of individuals with low antibody titer because any increase in the number of such individuals may cause an outbreak of measles.
The results of simulation indicated that the suppression of a measles outbreak requires the maintenance of high vaccine coverage, that a decline in vaccine coverage may cause a measles epidemic, and that the present routine immunization program of measles will maintain a low risk of an epidemic even after discontinuation of the third and fourth stages as scheduled-as long as at least $90 \%$ vaccine coverage of the first and second stages is maintained.

\section{Materials and methods}

\section{Study regions}

Okayama city, the capital of Okayama prefecture (population 674,746 in 2005 [21]), was almost equivalent in terms of vaccine coverage against measles to Japanese national average, and Kurashiki city in Okayama prefecture (population 469,377 in 2005 [21]) achieved high vaccine coverage in the second vaccination of measles [22, 23].

Okayama city and Kurashiki city were chosen as targeted regions in this study. The levels of measles vaccine coverage in Okayama city, Kurashiki city, and the mean coverage of the whole country in 2008 are shown in Table 1.

Progress in symptom of measles

The clinical symptoms of measles progress from catarrh (2-4 days) to rash (3-4 days), and then to a convalescence period (3-4 days) after a latent period of 8-12 days [24, 25]. Any severe complication, such as brain inflammation or pneumonia, may cause patient mortality. In this study, we adopted 5 days as the infection period and 10 days as the latent period, while we adopted 10 days as the infection period and 16 days as the latent period for modified measles patients $[24,25]$.

\section{Maternal immunity}

Maternal immunity declines approximately 6 months after birth on average [26]. A study on the antibody titer of

Table 1 Mean percentage of vaccination coverage (from April 2008 to March 2009)

\begin{tabular}{lllll}
\hline Location & \multicolumn{3}{l}{$\begin{array}{l}\text { Stage of routine immunization by measles } \\
\text { vaccination }\end{array}$} \\
\cline { 2 - 5 } & First (\%) & Second (\%) & Third (\%) & Fourth $(\%)$ \\
\hline Okayama city & 92.9 & 94.6 & 90.4 & 77.9 \\
Kurashiki city & 96.3 & 95.7 & 88.9 & 76.3 \\
Nationwide & 94.3 & 91.8 & 85.1 & 77.3 \\
\hline
\end{tabular}

Derived from National Institute of Infectious Diseases, 2008 [22, 23] 
children in Belgium [27] reported that (1) the maternal immunity of an infant born to a mother who had acquired immunity by vaccination was lower and also lost earlier than that of an infant born to a mother who had been infected with measles and (2) the loss of maternal immunity was not affected by the infant's weight, breast feeding, the educational level of the mother, or delivery by cesarean operation. In our study, the initial antibody titer of maternal immunity in an infant by the gelatin particle agglutination (PA) assay was assumed to be at one of two levels, i.e., $\mathrm{PA}=64$ or 128 , according to whether the maternal immunity derived from the mother acquired immunity by vaccination or by natural infection, respectively. The maternal immunity was also assumed to decline exponentially (Table 2).

Decline in the antibody titer over time

Prior to the introduction of mass immunization against measles, most people acquired measles immunity by natural infection [28]. However, in recent years, the majority have acquired measles immunity through vaccination, the antibody titer from which is lower than that acquired by natural infection. Owing the decline in antibody titer over time, the infection risk of measles should increase in youths who have been immunized by measles vaccination [27], which lead to a measles epidemic. On the other hand, both Christenson et al. [29] and Edmonson et al. [30] reported that the immunity acquired by natural infection also decreases in the long term, even though it is called lifetime immunity. It is generally considered that an antibody titer level of at least PA $\geq 128$-and preferably PA $\geq 256$ - is necessary to avoid being infected by the measles virus [31], while a titer level of PA $\leq 64$ conveys a risk of measles infection (modified measles). Therefore, we categorized recovered and vaccinated individuals into two subsets according to the antibody titer: $\mathrm{PA} \geq 128$ or $\mathrm{PA} \leq 64$.

Model for the transmission of measles

In this study, an age-time two-dimensional model for the transmission of measles was constructed. In this model, the steps of age and time are treated at daily intervals where age ranges from 0 to 100 years. The population is divided into several epidemiological classes and subclasses: susceptible (S), maternal immunity (M), exposed (E, Em; Em is the exposed class developing modified measles), infectious (I, Im; Im is the infectious class consisting of those with modified measles), recovered ( $\mathrm{R}, \mathrm{Rw}$; $\mathrm{Rw}$ is the recovered class with weak immunity), and vaccinated (V). An infant younger than 1.5 years of age who has never been infected with measles nor inoculated with measles vaccine belongs to the maternal immunity class or to the susceptible class according to his/her mother having immunity to measles or not, respectively; in addition, maternal immunity class is subdivided into maternal immunity derived from a vaccination (M1) and maternal immunity derived from natural infection (M2). When an infant loses maternal immunity, he/she moves to the S-class. The recovered class includes a subclass with weak immunity (Rw) in that the antibody titer starts to deteriorate $(\mathrm{PA} \leq 64)$. The vaccinated class is further classified into one of three subclasses: (1) V1, individuals who were vaccinated only once and have effective immunity (PA $\geq 128$ ); (2) V1w, individuals who were vaccinated once at a stage at which they still had maternal immunity or individuals with deteriorated antibody titer $(\mathrm{PA} \leq 64)$ acquired by a single vaccination; (3) V2, individuals who were vaccinated more than two times. The infection of an

Table 2 Assumed values of the model parameters

\begin{tabular}{lll}
\hline Description & Age (months) & Assumed value (per day) \\
\hline The rate of loss of maternal immunity & & 0.0122 \\
Mother acquired immunity by vaccination & $0-6$ & 0.0244 \\
& $7-18$ & 0.0063 \\
Mother acquired immunity by infection & $0-6$ & 0.0122 \\
& $7-18$ & Assumed value (per day) \\
\hline Description & Age (years) & $1.0 \times 10^{-3}$ \\
\hline The probability of coefficient of infection $(\beta)$ & $0-100$ & $5.56 \times 10^{-6}$ \\
The rate of loss of immunity acquired by infection & $20-60$ & $2.78 \times 10^{-6}$ \\
The rate of second vaccine failure (one-time vaccination) & $61-$ & $6.89 \times 10^{-5}$ \\
The proportion of primary vaccine failure & $1-10$ & $1.43 \times 10^{-5}$
\end{tabular}


individual belonging to the S-class with measles transfers him/her to the I-class through the E-class, while the infection of an individual belonging to V1w- or Rw-class with measles transfers him/her to the Im-class through Em. Recovery leads to transfer from the I- or Im-class to the R-class. The model scheme is shown in Fig. 1. The assessed values of the model parameters are shown in Table 2.

\section{Relative contact rate}

The force of infection at age $a$ is dependent on the contact rates with infectious individuals at age $a^{\prime}$ for all $a^{\prime}$. It is assumed that the contact rate among students in the same school year in primary and junior high schools is the highest and that among students in consecutive school years is the second highest, while that among adults or among adults and children is fairly low. We have assigned the relative contact rate $\operatorname{rc}\left(a, a^{\prime}\right)$ between individuals at ages $a$ and $a^{\prime}$ in comparison with the highest contact rate. Full details of relative contact rates are shown in Fig. 2. Therefore, the force of infection at age $a$ is given by the following formula:

$f(a)=\beta \int_{0}^{100} \operatorname{rc}\left(a, a^{\prime}\right)\left(\mathrm{I}\left(a^{\prime}\right)+\operatorname{Im}\left(a^{\prime}\right)\right) d a^{\prime}$

where $\beta$ is a probability coefficient of infection; the assumed value of $\beta$ refers to Table 2 .

\section{Scenario}

In order to analyze the influence of various vaccination coverages on the prevention of measles epidemic, we prepared several vaccination scenarios. As the standard level, we adopted the vaccination policy of Okayama city in 2008, consisting of vaccination coverage of one to four stages, which was similar to the average vaccination coverage nationwide [22, 23]. In baseline scenario 1 , a vaccination coverage of one to four stages is maintained as the standard level during 2008-2012. In scenario 2, the vaccination coverage of one to four stages is maintained in accordance with the vaccination coverage of one to four stages in Kurashiki city in 2008, which achieved a high coverage in the first and second stages in comparison with the nationwide average [22, 23]. We also prepared high and low levels of vaccination coverage in comparison with the standard level in scenarios 3 and 4 , respectively. To analyze the validity of the current vaccination policy in which the third and fourth stages are limited to 5 years (2008-2012), in scenarios 5-7, the vaccinations of the third and fourth stages are extended to 2018, and three levels of vaccination coverage are provided in these scenarios. All scenarios are summarized in Table 3. The population size for each scenario is fixed as the population of Okayama city in 2005, 674,746, to compare simulation results among scenarios easily.

\section{Results}

The age-time two-dimensional model was programmed by Intel Visual Fortran on Microsoft Visual Studio to work on any computer using the Microsoft Windows platform (Microsoft, Redwood, WA). In all scenarios, the initial values of epidemiological classes were determined on the basis of data on the age distribution of measles PA antibody positivity in Japan in 2006 [32] and coverage of measles immunization by age group (nationwide) in 2006 [33]; simulations proceed during $2006-2007$ by using the coverage of immunization in the respective year [34, 35]; thereafter, simulations proceed during 2008-2018 according to a scenario. For baseline scenario 1, the progression of the sectional distributions of epidemiological classes by
Fig. 1 Model scheme. For definition of epidemiological classes/subclasses refer to section Model for the transmission of measles

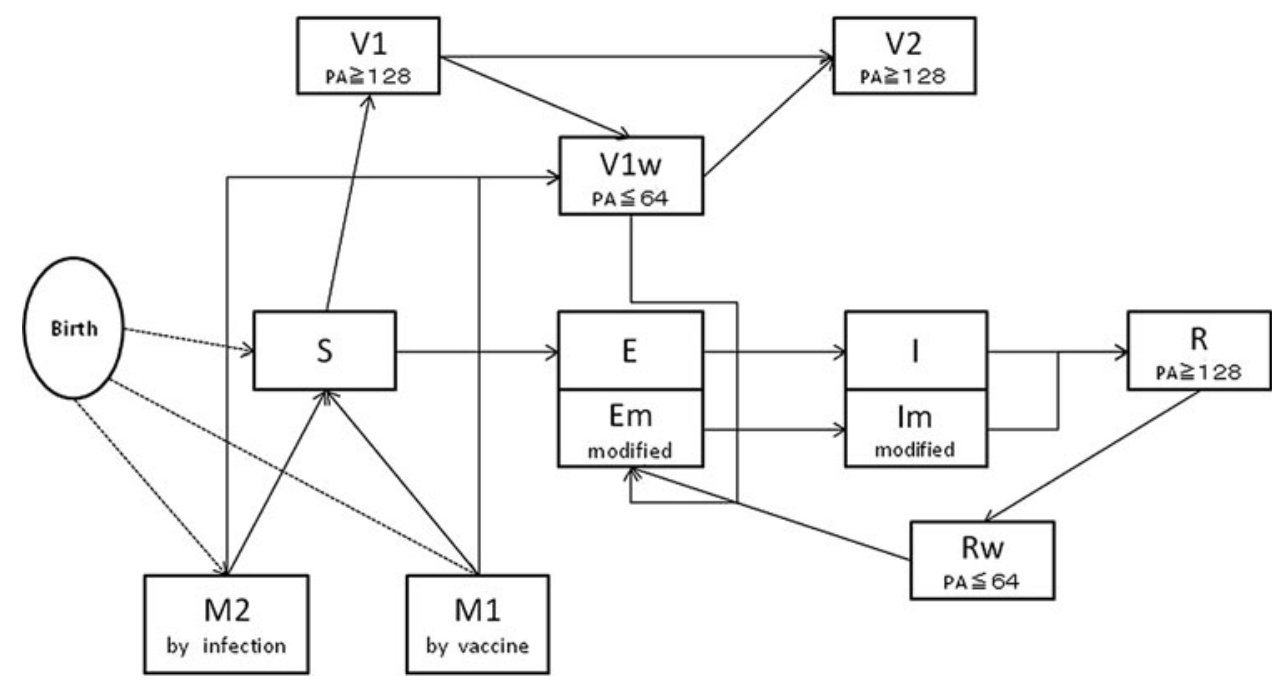


Fig. 2 Relative contact rates by age

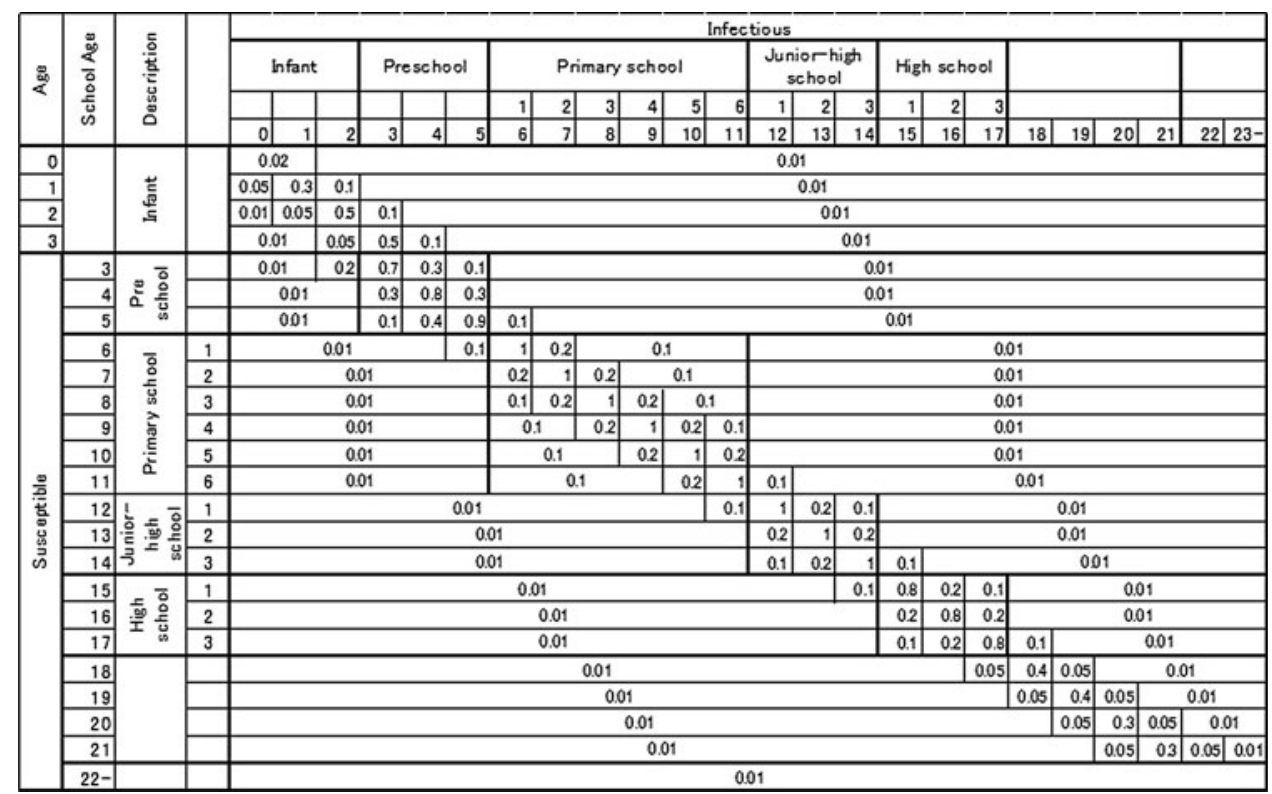

Table 3 Scenarios

\begin{tabular}{|c|c|c|c|c|c|}
\hline \multirow[t]{2}{*}{ Number of scenario } & \multirow[t]{2}{*}{ Scenario } & \multicolumn{4}{|c|}{ Stage of vaccination } \\
\hline & & First (\%) & Second $(\%)$ & Third $(\%)$ & Fourth $(\%)$ \\
\hline \multirow[t]{3}{*}{1.} & Okayama city & & & & \\
\hline & 2008-2012 & 92.9 & 94.6 & 90.4 & 77.9 \\
\hline & 2013-2018 & 92.9 & 94.6 & - & - \\
\hline \multirow[t]{3}{*}{2.} & Kurashiki city & & & & \\
\hline & 2008-2012 & 96.3 & 95.7 & 88.9 & 76.3 \\
\hline & 2013-2018 & 96.3 & 95.7 & - & - \\
\hline \multirow[t]{3}{*}{3.} & High level & & & & \\
\hline & 2008-2012 & 95.0 & 95.0 & 90.0 & 80.0 \\
\hline & 2013-2018 & 95.0 & 95.0 & - & - \\
\hline \multirow[t]{3}{*}{4.} & Low level & & & & \\
\hline & 2008-2012 & 90.0 & 90.0 & 80.0 & 70.0 \\
\hline & 2013-2018 & 90.0 & 90.0 & - & - \\
\hline \multirow[t]{3}{*}{5.} & Okayama city & & & & \\
\hline & 2008-2012 & 92.9 & 94.6 & 90.4 & 77.9 \\
\hline & 2013-2018 & 92.9 & 94.6 & 90.4 & 77.9 \\
\hline \multirow[t]{3}{*}{6.} & High level & & & & \\
\hline & 2008-2012 & 95.0 & 95.0 & 90.0 & 80.0 \\
\hline & 2013-2018 & 95.0 & 95.0 & 90.0 & 80.0 \\
\hline \multirow[t]{3}{*}{7.} & Low level & & & & \\
\hline & 2008-2012 & 90.0 & 90.0 & 80.0 & 70.0 \\
\hline & 2013-2018 & 90.0 & 90.0 & 80.0 & 70.0 \\
\hline
\end{tabular}

month-old age (0-100 years of age) in March in 2008, 2013, and 2018, which were obtained by the simulation, are shown in Fig. 3a, b, c, respectively. The proportions of susceptible and low antibody titer classes (S, Rw, V1w) in youths and young adults (aged 10-25 years) as of March in 2013 and 2018 were estimated as 5.9 and $3.4 \%$, respectively. The sectional distributions in Fig. 3d, e are limited to these three classes to show the details of the progression in the situation of youths and younger adults (<25 years old) as of March in 2013 and 2018, respectively.

We first compared scenario 2 , that is, a higher vaccination coverage situation in Kurashiki city in the first and 

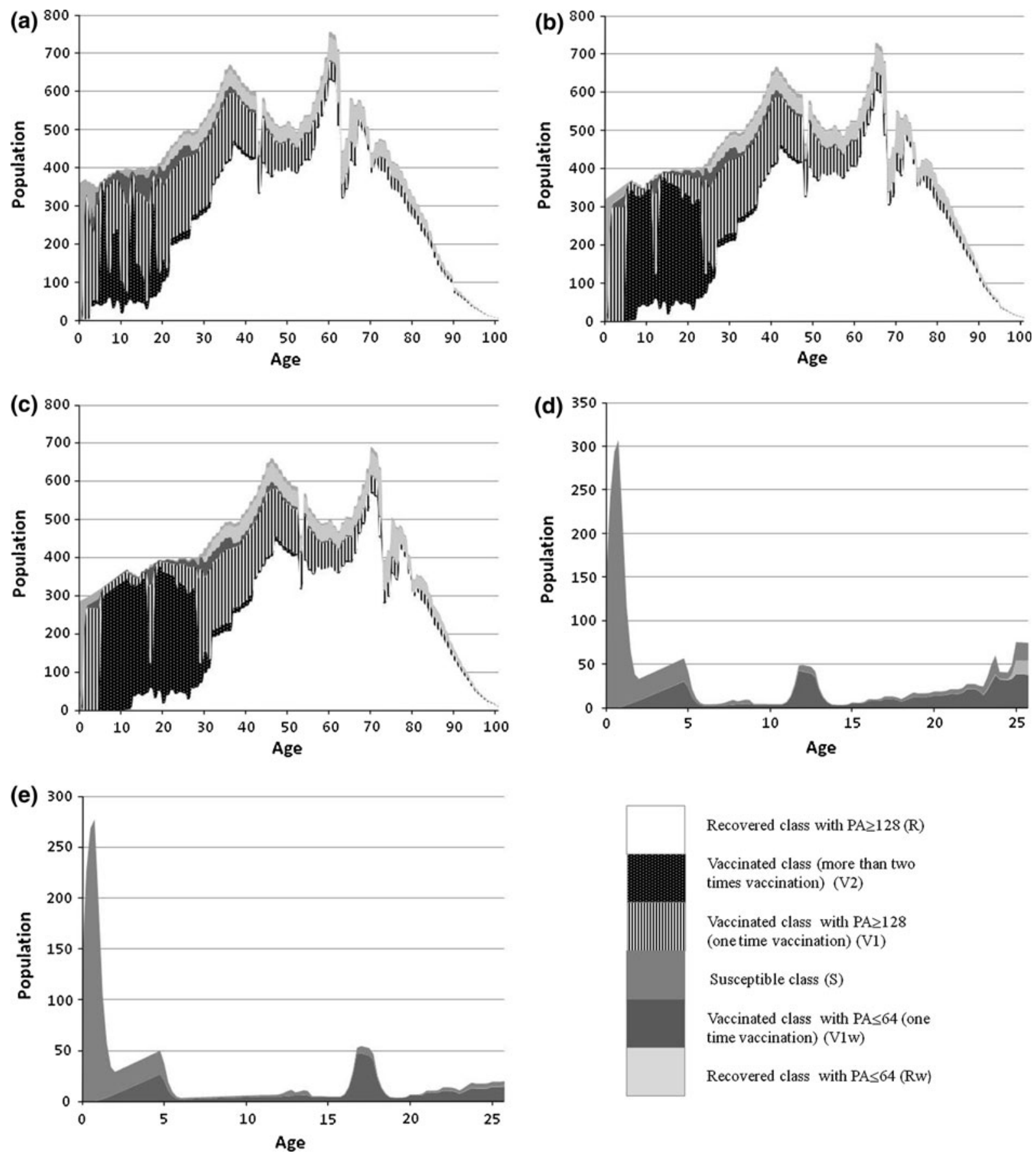

Fig. 3 The sectional distributions of epidemiological classes in March in scenario 1 (Okayama city). a 2008, b, d 2013, c, e 2018. d, e Limited to susceptible (S) and low antibody titer classes (Rw,

second stages of routine immunization, with baseline scenario 1 . According to the result of the simulation, the ratio of the total number of youths and young adults at an age of 10-25 as of March, 2013 who had no or insufficient immunity ( $\mathrm{S}, \mathrm{Rw}, \mathrm{V} 1 \mathrm{w}$ ) between scenarios 2 and 1 was 0.95:1, and the ratio as of March, 2018 fell to $0.57: 1$ (Fig. 4).

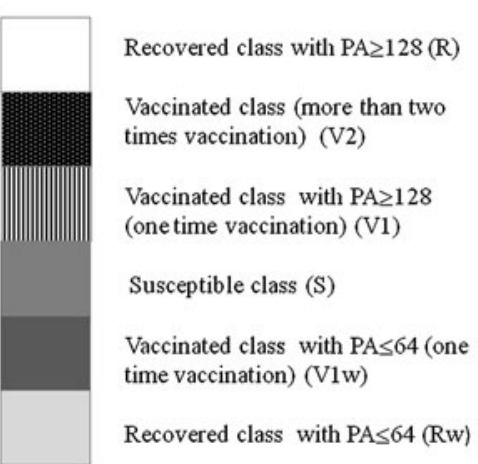

V1w). PA particle agglutination assay. The axis of ordinates shows the number of population by month-old age

We then prepared two scenarios, namely, 3 and 4, with high and low levels of vaccination coverage, respectively, where the third and the fourth stages of measles vaccination are discontinued in 2013. The aim of these scenarios was to examine how variation introduced in vaccination coverage would influence the number of individuals potentiality susceptible to measles virus infection. The 

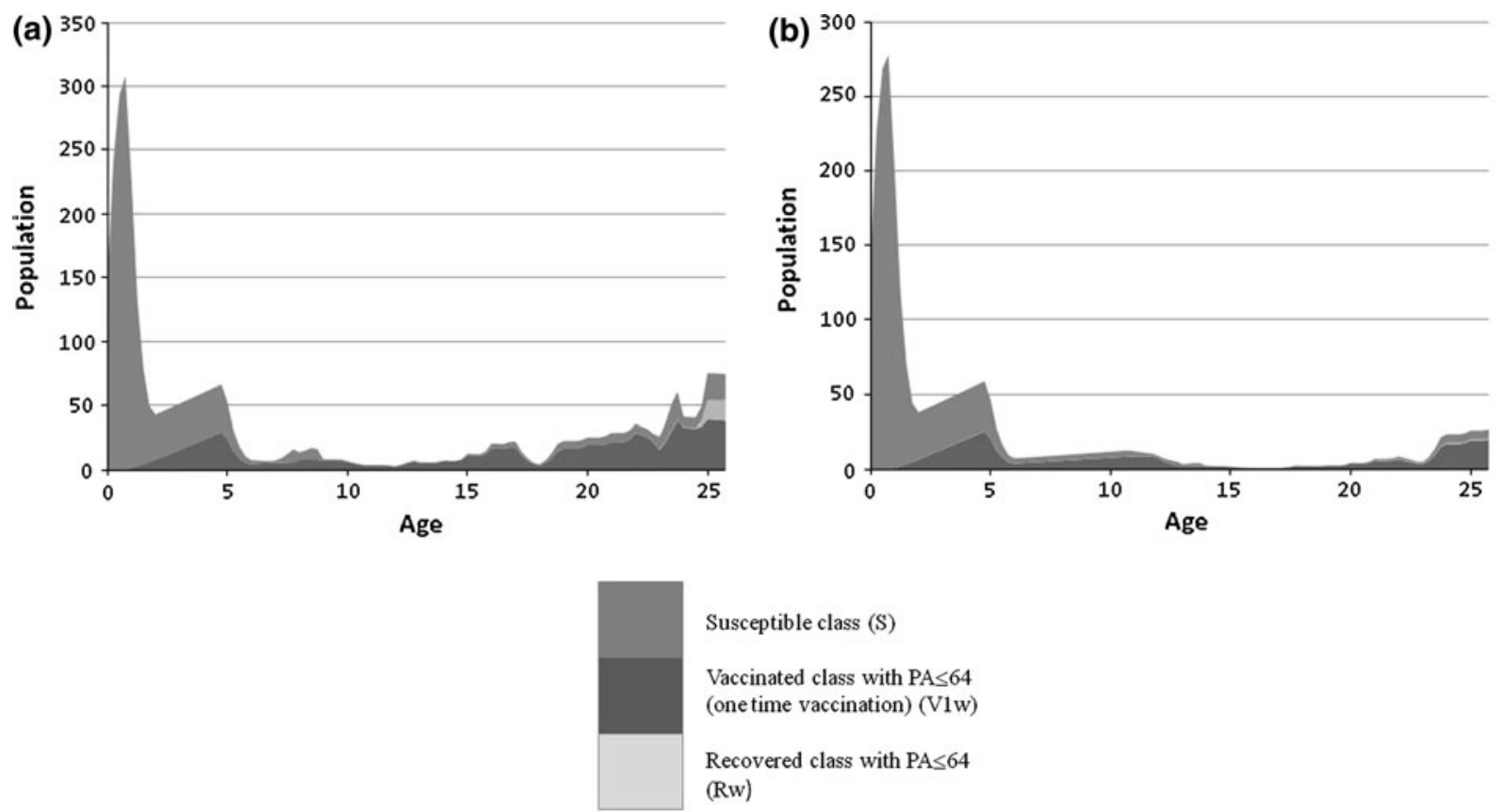

Fig. 4 The sectional distributions of three epidemiological classes (S, Rw, V1w) in March in scenario 2 (Kurashiki city). a 2013, b 2018. The axis of ordinates shows the number of population by month-old age

ratios for the total numbers of youths and young adults at an age of 10-25 years as of March, 2013 who had no or insufficient immunity (S, Rw, V1w) in scenarios 3 and 4 to that in baseline scenario 1 were assessed as $0.71: 1$ and 0.99:1, respectively (Fig. 5).

Finally, in scenarios 5-7, we extended the third and the fourth stages of measles vaccination until 2018. According to the results of the simulations, the proportions of individuals belonging to susceptible and low antibody titer classes (S, Rw, V1w) at an age 10-25 years as of March, 2018 , for scenarios 1,3 , and 4 with the third and fourth stages discontinued in 2013 were estimated at 3.4, 2.2, and $4.0 \%$, respectively, while those proportions for scenarios 5 , 6 , and 7 with the third and fourth stages extended until 2018 were estimated at very low levels of $1.3,0.76$, and $2.2 \%$, respectively. The progress of the sectional distributions of these three classes by month-old age as of March in 2018 for scenarios 6 and 7 are shown in Fig. 6a, b, respectively; the progression for scenario 5 is omitted because of its similarity to Fig. 6 a.

\section{Discussion}

In this study, we constructed an age-time two-dimensional model for the transmission of measles that was formulated by a system of partial differential equations. This model is able to describe the changes in numbers in epidemiological classes for both age and time in detail and can also precisely track changes in the vaccination schedule of measles so that a simulation of the model can assess the effect of various vaccination methods.

The maternal immunity class in infancy was introduced into the model. A vaccine at the first stage is unsuccessful during the period of preservation of maternal immunity [28]. On the other hand, an infant is faced with a risk of infection with the measles virus when his/her maternal immunity disappears. In addition, we distinguished maternal immunity as due to natural infection or vaccination in the course of the mother acquiring the immunity because maternal immunity derived from a mother obtained by vaccination is lower and lost earlier than that derived from a mother infected with measles [27, 28]. The introduction of this distinction in maternal immunity contributes to improving the accuracy of simulations in infants. In recent years, most mothers have acquired immunity by vaccination; therefore, this distinction has the additional advantage of the model automatically reflecting the relationship between the abundance of vaccinated mothers and the strength of maternal immunity in a long-term simulation.

Since the measles virus has a high infectivity, the basic reproduction number was estimated at high levels: 26.33 (Delhi, 1987), 12.76 (Kenya, 1984), 68.78 (Cameroon, 1975) [36] and so on [37]. We simply assigned the relative contact rate among individuals by age in comparison to the highest contact rate among students of the same school year in elementary and junior high schools. The value of 

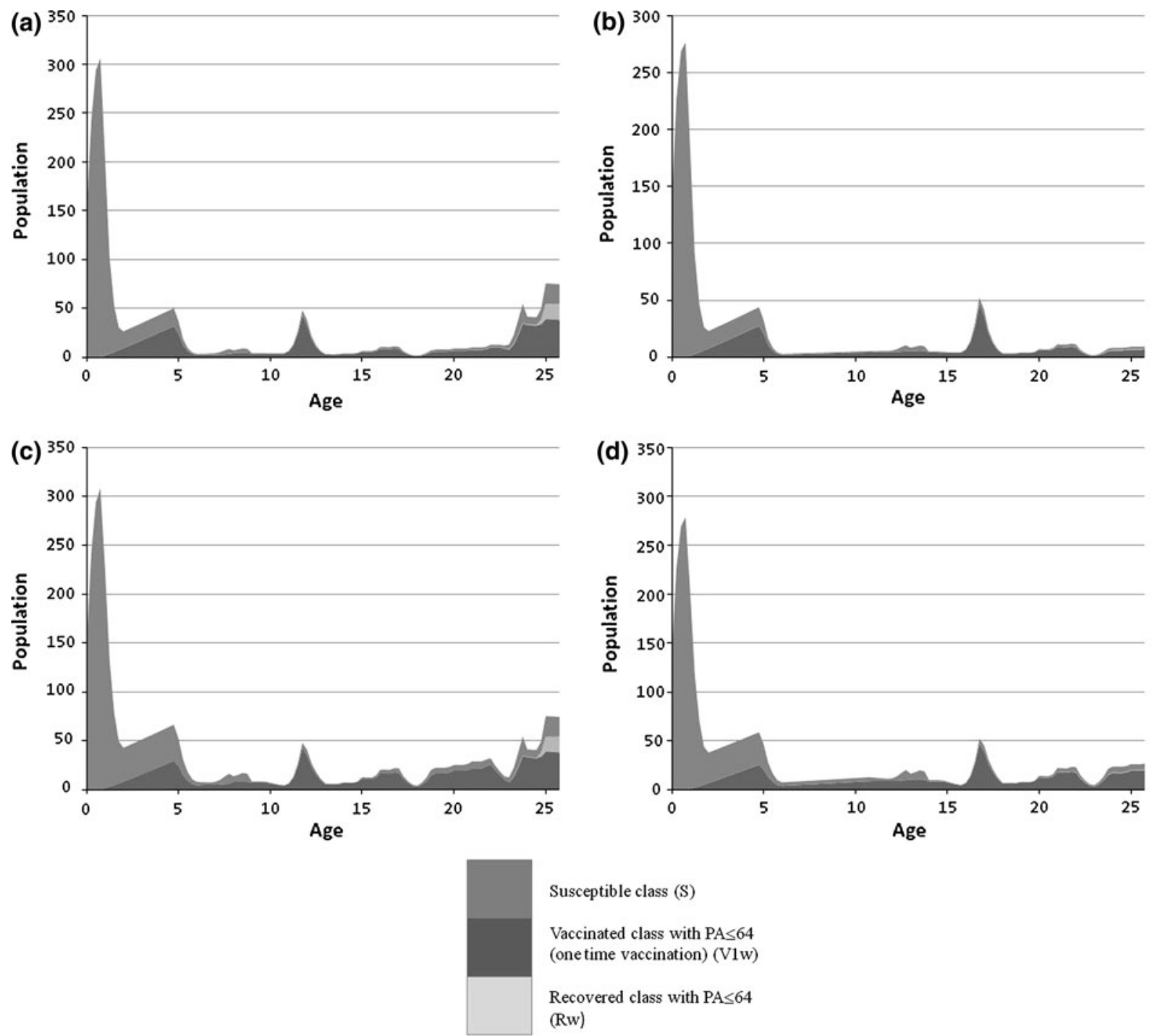

Fig. 5 The sectional distributions of epidemiological classes in March in scenarios 3 (a, b; high vaccination coverage) and 4 (c, d; low vaccination coverage). a, c 2013, b, d 2018. The axis of ordinates shows the number of population by month-old age

the probability coefficient of infection $(\beta)$ was chosen so that the model reflects an actual situation in terms of incidence. Further demographical studies on human behavior may contribute to the accuracy of the transmission model.

For a measles epidemic to be prevented, it is necessary to prevent an increase in the pool of individuals susceptible to the measles virus. Insufficient coverage of measles vaccination for several years in Japan is considered to be the cause of an outbreak in 2007 [14]. The result of our simulation showed that a high level of vaccine coverage (scenario 3) would lead directly to a reduction in the number of individuals who have no or low antibody titer, while the low level of vaccine coverage (scenario 4) would not lead to an increase in the number of such individuals, thereby indicating that the difference in the number of vaccinated individuals between the standard level (scenario 1) and the low level may correspond to the number of infections in scenario 4.

A considerable number of infants are left unvaccinated when there is only one opportunity for routine immunization against measles. A second failure of the one-time vaccination policy over time may increase the risk of infection with measles [29, 30]. On the occasion of the measles outbreak in 2007, students with a low antibody titer were found attending several colleges [38, 39]. A reinforced routine immunization program with the introduction of third and fourth stages, which was limited to a term of 5 years, had an effect on the suppression of measles. We examined the effect of the discontinuance of the third and fourth stages. For those scenarios based on the present routine immunization policy, the proportions of 

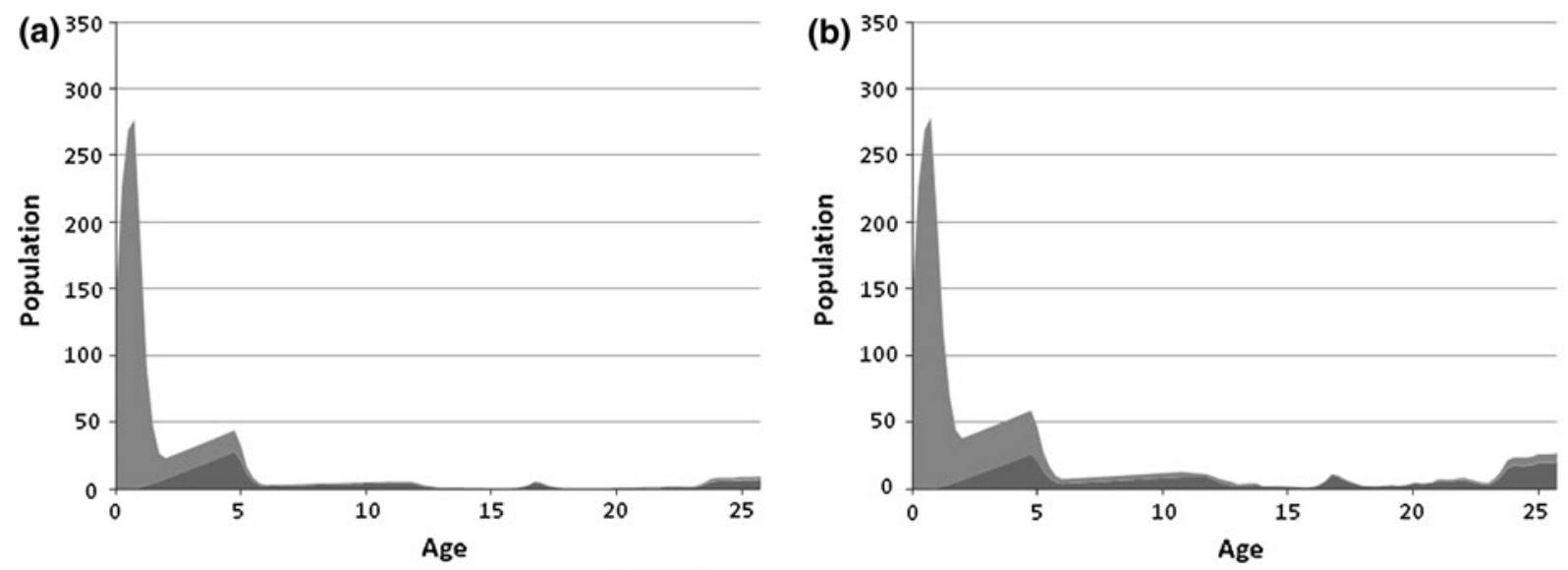

Susceptible class (S)

Vaccinated class with $\mathrm{PA} \leq 64$

(one time vaccination) (V1w)

Recovered class with PA $\leq 64$

(Rw)

Fig. 6 The sectional distributions of epidemiological classes in March 2018 in scenarios 6 (a; high vaccination coverage) and 7 (b; low vaccination coverage). The axis of ordinates shows the number of population by month-old age

individuals belonging to susceptibly low antibody titer classes in 2018 at an age of 10-25 years decreased to $2-3 \%$ in scenarios 1 and 3 , or the proportion was maintained at $4 \%$ in scenario 4 , while for scenarios $5-7$ in which the third and fourth stages were extended, the proportions decreased to $1-2 \%$. The results of this quantitative study indicate that the suppression of measles outbreak requires the maintenance of high vaccine coverage and that a decline in vaccine coverage may result in a measles epidemic.

The present routine immunization program of measles will maintain a low risk of an epidemic after the discontinuation of the third and fourth stages as scheduled, as long as at least $90 \%$ vaccine coverage of the first and second stages is maintained. Therefore, the simulation results support discontinuation of the third and fourth stages of vaccination as scheduled so long as endeavors are continued to maintain appropriate high vaccine coverage of the first and second stages.

Among the developed countries Japan has a relatively high incidence of measles, and it is also held responsible for the export of measles to measles-free countries. Therefore, it is important to implement a measles-elimination program at the national level.

Acknowledgments This work was supported in part by a Grant-inAid from the Japan Society for the Promotion of Science (21540129) and a Grant-in-Aid from the Ministry of Health, Labour and Welfare of Japan (H20-Sinkou-ippan-013).

Conflict of interest There are no conflicts of interest.

\section{References}

1. Infectious Disease Surveillance Center. Measles. 2002. Available at: http://idsc.nih.go.jp/disease/measles/report2002/measles_top. html\#mashin01 (in Japanese).

2. Infectious Disease Surveillance Center. Immunization schedule. 2010. Available at: http://idsc.nih.go.jp/vaccine/dschedule/Imm 10-03JP.gif (in Japanese).

3. Niigata-city. Efforts toward high second dose measles-rubella vaccine coverage (in Japanese). Infect Dis Wkly Rep Jpn. 2008;10(31):17-9. Available at: http://idsc.nih.go.jp/disease/mea sles/sokuhoumeas/0831.pdf.

4. Odawara-city. Efforts toward second dose vaccine encouragement of measles-rubella vaccination. Infect Dis Wkly Rep Jpn. 2008;10(30):16-7 (in Japanese). Available at: http://idsc.nih.go. jp/disease/measles/sokuhoumeas/0830.pdf.

5. Takamatsu-city. Efforts toward high second dose measles-rubella vaccination coverage in 2007 (in Japanese). Infect Dis Wkly Rep Jpn. 2008;10(28):20-1. Available at: http://idsc.nih.go.jp/disease/ measles/sokuhoumeas/0828.pdf).

6. Center of Public Health, Kurashiki-city. Efforts toward high second dose measles-rubella vaccine coverage (in Japanese). Infect Dis Wkly Rep Jpn. 2008;10(27):20-1. Available at: http:// idsc.nih.go.jp/disease/measles/sokuhoumeas/0827.pdf.

7. Fukui-prefecture. Factor for attaining a high measles-rubella vaccine coverage in preschoolers (6-7 years) in fiscal year 2007Fukui (in Japanese). Infect Dis Wkly Rep Jpn. 2008;10(26): 13-4. Available at: http://idsc.nih.go.jp/disease/measles/ sokuhoumeas/0826.pdf.

8. Akita-prefecture. Efforts toward high measles-rubella (MR) vaccine coverage $(>95 \%)$ in preschoolers $(6-7$ years) in fiscal year 2007-Akita (in Japanese). Infect Dis Wkly Rep Jpn. 2008;10(25):15-6. Available at: http://idsc.nih.go.jp/disease/mea sles/sokuhoumeas/0825.pdf.

9. Johetsu-city. Efforts toward high third and forth measles-rubella vaccine coverage in Johetsu in 2008 (in Japanese). Infect Dis 
Wkly Rep Jpn. 2009;11(11):18-20. Available at: http://idsc.nih. go.jp/disease/measles/sokuhoumeas/0911.pdf.

10. Sakura-Center of Public Health, Tsukuba-city. Efforts toward second dose measles-rubella vaccine coverage in junior high school students (in Japanese). Infect Dis Wkly Rep Jpn. 2009;11(10):14-5. Available at: http://idsc.nih.go.jp/disease/mea sles/sokuhoumeas/0910.pdf.

11. Center of Public Health, Hakodate-city. Efforts toward high third and forth dose measles-rubella vaccine coverage in junior high and high school students in 2008 (in Japanese). Infect Dis Wkly Rep Jpn. 2009;11(7):18-20. Available at: http://idsc.nih.go.jp/ disease/measles/sokuhoumeas/0907.pdf.

12. Center of Public Health, Hamamatsu-city. Efforts toward high second dose measles-rubella vaccine coverage in junior high and high school students-Hamamatsu City (in Japanese). Infect Dis Wkly Rep Jpn. 2009;11(3):23-4. Available at: http://idsc.nih. go.jp/disease/measles/sokuhoumeas/0903.pdf.

13. Lee K, Kim H, Shin E, Kim Y, Chang S, Choi J. Validity of measles immunization certificates submitted upon enrollment in an elementary school in Korea. J Prev Med Public Health. 2009;42:104-8

14. Nagai M, Xin JY, Yoshida N, Miyata A, Fujino M, Ihara T, et al. Modified adult measles in outbreak in Japan, 2007-2008. J Med Virol. 2009;81:1094-101.

15. Paulo AC, Gomes MC, Gomes MG. Dynamics and control of measles in Portugal: accessing the impact of anticipating the age for the first dose of MMR from 15 to 12 months of age. Vaccine. 2008;26:2418-27.

16. Bauch CT, Earn DJ. Transients and attractors in epidemics. Proc Biol Sci. 2003;270:1573-8.

17. Schenzle D. An age-structured model of pre- and post-vaccination measles transmission. IMA J Math Appl Med Biol. 1984;1:169-91.

18. Manfredi P, Williams JR, Ciofi Degli Ati ML, Salmaso S. Measles elimination in Italy: projected impact of the National Elimination Plan. Epidemiol Infect. 2005;133:87-97.

19. Roberts MG, Tobias MI. Predicting and preventing measles epidemics in New Zealand: application of a mathematical model. Epidemiol Infect. 2000;124:279-87.

20. Eicner M, Zehnder S, Diets K. An age-structured model for measles vaccination. In: Isham V, Medley G, editors. Models for infectious human diseases: their structure and relation to data. Cambridge: Cambridge University Press; 1996. p. 38-56.

21. Ministry of Internal Affairs and Communications Bureau of Statistics of Japan. National census (in Japanese). 2005. http://www.stat.go.jp/data/kokusei/2005/index.htm.

22. Infectious Disease Surveillance Center. National survey on measles-rubella vaccine coverage in Japan-final results in 2008. Infectious Agents Surveillance Report. 2010;31:39-40. Available at: http://idsc.nih.go.jp/iasr/31/360/dj3603.html.

23. Infectious Disease Surveillance Center. National survey on measles-rubella vaccine coverage in Japan-final results in 2008, 2009 (in Japanese). Available at: http://idsc.nih.go.jp/disease/mea sles/pdf02/20090812-01.pdf, http://idsc.nih.go.jp/disease/measles/ pdf02/20091105-01.pdf; http://idsc.nih.go.jp/disease/measles/pdf 02/20091105-02.pdf; http://idsc.nih.go.jp/disease/measles/pdf02/ 20091105-03.pdf; http://idsc.nih.go.jp/disease/measles/pdf02/2009 1105-04.pdf.

24. Cherry JD. Measles virus. In: Feigin RD, Cherry JD, editors. Textbook of pediatric infectious diseases. New York: WB Saunders; 1998.

25. Katz SL. Measles (Rubeola). In: Katz SL, Gershon AA, Hotez PJ, editors. Krugman's infectious diseases of children, 10th edn. Maryland Heights: Mosby-Year Book; 1998.

26. Eichner M, Diebner HH, Schubert C, Kreth HW, Dietz K. Estimation of the time-dependent vaccine efficacy from a measles epidemic. Stat Med. 2002;21:2355-68.

27. Leuridan E, Hens N, Hutse V, Ieven M, Aerts M, Damme VP. Early waning of maternal measles antibodies in era of measles elimination: longitudinal study. Br Med J. 2010;340:c1626.

28. Leuridan E, Damme VP. Passive transmission and persistence of naturally acquired or vaccine-induced maternal antibodies against measles in newborn. Vaccine. 2007;25:6296-304.

29. Christenson B, Bottiger M. Measles antibody: comparison of long-term vaccination titres, early vaccination titres and naturally acquired immunity to and booster effects on the measles virus. Vaccine. 1994;12:129-33.

30. Edmonson MB, Addiss DG, McPherson JT, Berg JL, Circo SR, Davis JP. Mild measles and secondary vaccine failure during a sustained outbreak in a highly vaccinated population. JAMA. 1990;263:2467-71.

31. Taya S, Satoh H, Kitamoto R, Okabe N. Measles antibody prevalence rate and vaccine coverage in Japan, 2008-NESVPD (in Japanese). Infect Agents Surveillance Rep. 2009;30:40-3. Available at: http://idsc.nih.go.jp/iasr/30/348/dj3486.html.

32. Infectious Disease Surveillance Center. Age distribution of measles PA antibody positives in Japan. 2006. Available at: http://idsc.nih.go.jp/yosoku/Measles/Serum-M2006.html.

33. Infectious Disease Surveillance Center. Coverage of measles immunization by age/age group. 2006. Available at: http:// idsc.nih.go.jp/vaccine/atopics/meas070406.gif.

34. Infectious Disease Surveillance Center. National epidemiological surveillance of vaccine-preventable diseases. 2006. Available at: http://idsc.nih.go.jp/yosoku/Measles/Vac-M2006-e.html.

35. Infectious Disease Surveillance Center. National epidemiological surveillance of vaccine-preventable diseases. 2007. Available at: http://idsc.nih.go.jp/yosoku/Measles/Vac-M2007-e.html.

36. Szusz EK, Garrison LP, Bauch CT. A review of data needed to parameterize a dynamic model of measles in developing countries. BMC Res Notes. 2010;3:75.

37. Anderson RM, May RM. Infectious diseases of humans dynamics and control. Oxford: Oxford University Press; 1991.

38. Soka University. Response to measles outbreak at Soka University, April-May 2007-Tokyo (in Japanese). Infect Agents Surveillance Rep. 2007;28:247-9. Available at: http://idsc.nih.go.jp/ iasr/28/331/dj3313.html.

39. Otsuma women's University. Measles among women's university students: countermeasures utilizing maternity and child health record books (in Japanese). Infect Agents Surveillance Rep. 2007;28:250. Available at: http://idsc.nih.go.jp/iasr/28/331/ dj3315.html. 